\title{
Cost effectiveness analysis of antenatal HIV screening in United Kingdom
}

\author{
A E Ades, M J Sculpher, D M Gibb, R Gupta, J Ratcliffe
}

Editorial by
Peckham
Department of
Epidemiology and
Public Health,
Institute of Child
Health, London
WC1N 1EH
A E Ades
reader
D M Gibb
senior lecturer in
epidemiology
R Gupta
research assistant
Centre for Health
Economics, York
University,
Heslington, York
YO10 5DD
M J Sculpher
senior research fellow
Health Economics
Research Group,
Brunel University,
Uxbridge,
Middlesex
UB8 3PH
J Ratcliffe
research fellow
Correspondence to:
A E Ades
a.ades@ich.ucl.ac.uk

BMJ 1999;319:1230-4

\begin{abstract}
Objective To assess the cost effectiveness of universal antenatal HIV screening compared with selective screening in the United Kingdom.

Design Incremental cost effectiveness analysis relating additional costs of screening to life years gained. Maternal and paediatric costs and life years were combined.

Setting United Kingdom.

Main outcome measures Number of districts for which universal screening would be cost effective compared with selective screening under various conditions.

Results On base case assumptions, a new diagnosis of a pregnant woman with HIV results in a gain of 6.392 life years and additional expenditure of $£ 14$ 833. If decision makers are prepared to pay up to $£ 10000$ for an additional life year, this would imply a net benefit of $£ 49090$ (range $£ 12$ 300-£59 000), which would be available to detect each additional infected woman in an antenatal screening programme. In London, universal antenatal screening would be cost effective compared with a selective screening under any reasonable assumptions about screening costs. Outside London, universal screening with uptake above $90 \%$ would be cost effective with a $£ 0.60 \mathrm{HIV}$ antibody test cost and up to 3.5 minutes for pretest discussion. Cost effectiveness of universal testing is lower if selective testing can achieve high uptake among those at higher risk. A universal strategy with only $50 \%$ uptake may not be less cost effective in low prevalence districts and may cost more and be less effective than a well run selective strategy.

Conclusions Universal screening with pretest discussion should be adopted throughout the United Kingdom as part of routine antenatal care as long as test costs can be kept low and uptake high.
\end{abstract}

\section{Introduction}

Each year in the United Kingdom there are over 300 births to women infected with HIV, but over $75 \%$ of these maternal infections remain undiagnosed at the time of birth. ${ }^{1}$ Evidence that antiretroviral drugs and elective caesarean delivery reduce the risk of vertical transmission has established a clinical rationale for antenatal testing. ${ }^{2}{ }^{3}$ Earlier Department of Health guidelines called for HIV testing to be offered to all pregnant women in areas of higher prevalence and to those at high risk elsewhere. ${ }^{4}$ However, a policy of universal testing throughout the United Kingdom has recently been announced. ${ }^{5}$ This paper presents a cost effectiveness analysis comparing universal and selective antenatal HIV testing. A full technical report of the study is available. ${ }^{6}$

\section{Methods}

Net benefit of diagnosing maternal HIV infection

The cost effectiveness of any programme of antenatal testing depends on the additional costs (or savings) that result from diagnosis of maternal HIV infection and the life years gained. Earlier diagnosis of HIV in the mother generates additional costs but also increases her life expectancy. ${ }^{7}$ Interventions that reduce the risk of vertical transmission both avert the lifetime costs of caring for an HIV infected child and gain life years. However, not all vertical transmission is prevented, and infected children followed from birth can be expected to have higher lifetime care costs, but also longer life expectancy, than infected children born to mothers whose infection was not recognised in pregnancy. ${ }^{8}$

Based on the uptake of elective caesarean section and zidovudine among infected pregnant women in 1997, maternal diagnosis would reduce vertical transmission rates from $26.5 \%$ to $7.6 \% .{ }^{19}$ We also explored the effect of $100 \%$ uptake of both interventions, with an estimated transmission rate of $3.8 \%{ }^{9}$ A fifth of infected women diagnosed antenatally elect for termination of pregnancy. ${ }^{1}$ Although the costs of termination are included in the analysis, the life years lost and lifetime care costs avoided are not included. ${ }^{6}$

We combined paediatric and maternal costs and life years and expressed them in terms of net benefit of maternal diagnosis. ${ }^{10}$ If a decision maker is willing to pay $£ 10000$ per additional life year, based on the costs and effects of other interventions funded by the NHS, ${ }^{11}$ the net benefit of diagnosis is $£ 10000$ multiplied by the number of life years gained minus the additional downstream costs. The net benefit is the amount that it would be cost effective to spend in order to identify one additional infected woman. Table 1 lists all the cost, life year, and probability parameters arising after maternal diagnosis.

Each of the determinants of net benefit is subject to multiple sources of uncertainty and a full sensitivity analysis is available. ${ }^{6}$ Here we present the maximum and minimum net benefit estimates. These define the most optimistic and most pessimistic conditions for antenatal testing.

Costs of antenatal screening and epidemiology of undiagnosed HIV infection

Table 2 lists the factors influencing the costs and effectiveness of the screening programme before maternal diagnosis. Our analysis compares a universal strategy offering HIV testing to all pregnant women with a selective strategy offering testing to those at high risk of infection-that is, injecting drug users, black African women, women who have previously attended sexually transmitted disease clinics, and women with multiple or known high risk partners. Costs for the selective strategy do not include additional time identifying women at higher risk. A quarter of women at higher risk and 5\% at low risk are assumed to request screening even in the 
Table 1 Factors that determine the net benefit of antenatal diagnosis: base case values and alternative values for sensitivity analysis. Costs (£s) are discounted at $6 \%$ a year. Estimated life years discounted at $2 \%$ a year are shown in parenthesis

\begin{tabular}{|c|c|c|}
\hline & Base case value & Alternative values \\
\hline \multicolumn{3}{|l|}{ Management of women during pregnancy } \\
\hline Probability of termination if HIV detected during pregnancy ${ }^{1}$ & $20 \%$ & $0 \%-25 \%$ \\
\hline Cost of termination ${ }^{6}$ & 444 & $300-600$ \\
\hline Cost of initial counselling and management of HIV positive woman during pregnancy ${ }^{6}$ & 69 & $58-174$ \\
\hline Cost of additional medical management of woman with HIV during pregnancy ${ }^{6}$ & 306 & $118-670$ \\
\hline Cost of "normal" birth" & 467 & $300-600$ \\
\hline \multicolumn{3}{|l|}{ Interventions to reduce risk of vertical transmission } \\
\hline Cost of risk reduction strategy (including delivery) ${ }^{\star 9}$ & 1412 & 1189-1968 \\
\hline Rate of vertical transmission without risk reduction strategies ${ }^{9}$ & $26.5 \%$ & $21.1 \%-31.9 \% \dagger$ \\
\hline Rate of vertical transmission with risk reduction strategies ${ }^{9}$ & $7.6 \%$ & $1.7 \%-12.7 \% \dagger$ \\
\hline \multicolumn{3}{|l|}{ Paediatric costs and life expectancy } \\
\hline Cost of diagnosing uninfected child, mother's HIV diagnosed during pregnancy ${ }^{12}$ & 512 & $246-981$ \\
\hline Lifetime cost of HIV infected child, mother's infection identified during pregnancy $\ddagger^{8}$ & 73855 & $24197-215077$ \\
\hline Lifetime cost of HIV infected child, mother's infection not known during pregnancy $\ddagger^{8}$ & 59004 & $20174-142568$ \\
\hline Life expectancy of HIV infected child, mother's infection not known during pregnancy (years) $\ddagger^{8}$ & $10.09(8.60)$ & $5.94-18.54(5.39-14.36)$ \\
\hline Life expectancy of HIV infected child, mother's infection identified during pregnancy (years) $\ddagger^{8}$ & $11.66(9.87)$ & $6.32-23.81(5.74-8.06)$ \\
\hline Life expectancy of child who is not infected (years) ${ }^{13}$ & $77(39.51)$ & $73-79$ (38.59-39.93) \\
\hline \multicolumn{3}{|l|}{ Mother's costs after pregnancy and life expectancy } \\
\hline Cost of managing woman with HIV whose infection was diagnosed during pregnancy $\S^{7}$ & 102812 & $88075-155192$ \\
\hline Cost of managing woman with HIV whose infection was not known during pregnancy $\S^{7}$ & 79494 & $74100-83067$ \\
\hline Life expectancy of HIV infected woman whose infection was diagnosed during pregnancy (years) $\S^{7}$ & $18.25(13.96)$ & 15.41-25.48 (12.0-8.43) \\
\hline Life expectancy of HIV infected woman whose infection was not diagnosed during pregnancy (years) $\S^{7}$ & $17.63(13.50)$ & 15.28-20.88 (11.9-15.5) \\
\hline Maximum willingness to pay per life year gained ${ }^{11}$ & 10000 & $6000-20000$ \\
\hline
\end{tabular}

${ }^{*}$ Model based on observed uptake of interventions in United Kingdom 1997 (75\% zidovudine, 44\% caesarean section, 96\% bottle feeding). ${ }^{19}$ Minimum assumes 6\% caesarean section rate resulting in transmission rate of $8.8 \%$; maximum assumes $100 \%$ uptake of interventions and $3.8 \%$ vertical transmission rate. $^{6} 9$ $+95 \%$ confidence interval.

$\ddagger$ Base case scenario: moderate resource use, moderate efficacy of antiretroviral therapy (0.67) and prognostic variables; antiretroviral therapy started two years before AIDS. Minimum assumes: low resource use, pessimistic antiretroviral efficacy $(0.8)$ and prognostic variables; antiretrovirals started at onset of AIDS. Maximum assumes: high resource use, optimistic efficacy $(0.6)$ and prognostic variables; antiretrovirals started at birth or diagnosis. ${ }^{6} 8$

$\S$ Base case assumes moderate efficacy of antiretroviral therapy (0.6); antiretrovirals started at CD4 count $350 \times 10^{6} / /$; unscreened woman diagnosed 20 months after booking. Minimum additional cost per life year gained between screened and unscreened: low antiretroviral efficacy (0.80); antiretrovirals started at CD4 count $350 \times 10^{6} /$; unscreened woman diagnosed 10 months after booking. Maximum additional cost per life year gained between screened and unscreened: high antiretroviral efficacy (0.40); antiretrovirals started at diagnosis; unscreened woman diagnosed 51 months after booking. ${ }^{6}{ }^{7}$

absence of a formal selective or universal strategy. Uptake among the higher risk groups under a selective strategy is assumed to be $20 \%$ in the base case analysis (that is, $20 \%$ of those who would not anyway be tested on request) with an upper limit of $60 \%$. Universal testing was assumed to have $95 \%$ coverage, although $50 \%$ and $90 \%$ uptake were also examined.

We used a decision model ${ }^{6}$ to generate prevalence thresholds above which universal screening would be cost effective compared with selective screening. These thresholds were compared with estimates of the prevalence of undiagnosed HIV infection in $\mathrm{p}$ regnant women in British health authorities (fig 1), to determine how many districts should adopt a universal strategy under different assumptions. The prevalence of undiagnosed infection has been estimated elsewhere. ${ }^{6}{ }^{14}$

Costing is based on a health service perspective at 1996-7 prices. We discounted costs and life years using annual discount rates of $6 \%$ and $2 \%$, respectively, following Department of Health guidelines. ${ }^{15}$ The decision model equations are detailed elsewhere. ${ }^{6}$

\section{Results}

Net benefit of antenatal diagnosis of HIV

On base case assumptions, maternal diagnosis leads to an additional £24 383 lifetime cost in caring for the mother and an additional 0.455 years of life for the mother (table 3). To prevent one paediatric infection, it is necessary to diagnose about 5.3 infected women. On average one maternal diagnosis saves $£ 9550$ in the costs of caring for children and gains 5.937 child life years. Overall, therefore, maternal diagnosis results in additional downstream costs of $£ 14833$ $(£ 24383-£ 9550)$ and results in a gain of 6.392 $(0.455+5.937)$ life years.

For a decision maker willing to pay up to $£ 10000$ to gain one life year, the net benefit of diagnosis would be $£ 49090((6.392 \times £ 10000)-£ 14833))$, and it would be cost effective to devote this sum to detect one additional infected woman in an antenatal testing programme. The most pessimistic estimate of net benefit was $£ 12335$ and the most optimistic $£ 59012$.

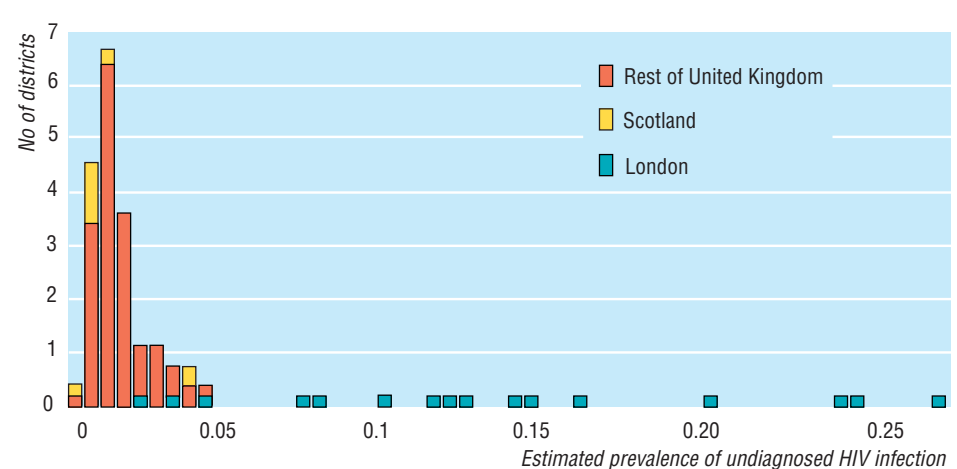

Fig 1 Frequency distribution of prevalence of undiagnosed HIV in pregnant women in London, Scotland, and rest of United Kingdom, 1993-6. Estimates are based on data from unlinked anonymous neonatal surveys regressed against data on prevalence of treated infection and also take into account data on extent of clinical diagnosis before antenata attendance $^{6}$ 
Table 2 Factors affecting antenatal screening programme: base case and minimum and maximum values

\begin{tabular}{|c|c|c|}
\hline & $\begin{array}{l}\text { Base case } \\
\text { value }\end{array}$ & $\begin{array}{c}\text { Minimum anc } \\
\text { maximum } \\
\text { values }\end{array}$ \\
\hline Cost of test $(£)$ & 2.70 & $0.60,15.00$ \\
\hline Average cost of offer and pretest discussion $(£)^{6 *}$ & 0 & $0,1.50$ \\
\hline $\begin{array}{l}\text { False negative rate (initial screen), and false positive rate of test } \\
\text { algorithm }{ }^{6}\end{array}$ & 0 & \\
\hline Uptake of testing in higher risk groups with "on request" policy & 0.25 & \\
\hline Uptake of testing in low risk group with "on request" policy & 0.05 & \\
\hline Uptake of testing in higher risk groups with selective testing $\dagger$ & 0.20 & 0.60 \\
\hline Uptake of testing with universal policy ${ }^{5}$ & 0.95 & $0.50,0.90$ \\
\hline \multicolumn{3}{|l|}{ Average percentage of antenatal population in higher risk groups $\neq \S^{6}{ }^{14}$ : } \\
\hline London & 14.95 & \\
\hline Outside London & 5.55 & \\
\hline \multicolumn{3}{|l|}{ Prevalence of undiagnosed HIV in higher risk groups $(\%) \ddagger^{6}{ }^{14}$ : } \\
\hline London & 0.98 & \\
\hline Outside London & 0.18 & \\
\hline \multicolumn{3}{|l|}{ Prevalence of undiagnosed HIV in low risk groups $(\%) \ddagger^{6}{ }^{14}$ : } \\
\hline London & 0.014 & \\
\hline Outside London & 0.002 & \\
\hline \multicolumn{3}{|l|}{ Average prevalence (range) of undiagnosed infection in UK districts $(\%) \ddagger^{6}$ : } \\
\hline London & 0.16 & $0.021-0.28$ \\
\hline Outside London & 0.013 & $0.0021-0.038$ \\
\hline
\end{tabular}

${ }^{\star} £ 0.25$ per minute.

tRefers to those not tested on request. Thus base case net uptake for selective screening =

$0.25+(0.75 \times 0.20)=0.40$

tData from Scotland and England and Wales outside London are very similar ${ }^{6}$ and have been combined.

$\S$ District prevalence is assumed to vary within regions because of variation in the proportion at higher risk. Results obtained with alternative assumptions are presented elsewhere. ${ }^{6}$

We have estimated elsewhere that an infected woman not diagnosed in pregnancy will be diagnosed on average 20 months later (range 12 to 51 months). ${ }^{67}$ Net benefit was highly sensitive to this parameter. It was also sensitive to the resources required to care for an HIV infected child because higher paediatric care costs mean that preventing vertical transmission produces greater savings. Net benefit was relatively insensitive to efficacy of antiretroviral treatment in delaying disease progression because more effective treatment generates additional life years in approximate proportion to the increased costs incurred.

Net benefit depends on the maximum decision makers are willing to spend to gain one additional life year (figure 2). Given a $£ 10000$ willingness to pay per

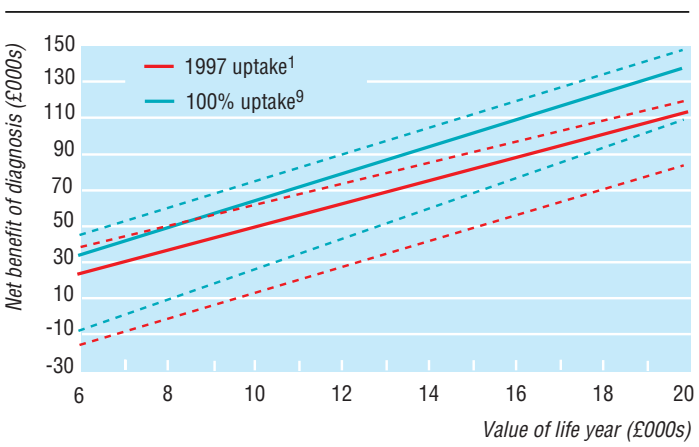

Fig 2 Net benefits of screening in relation to value of an additiona life year. Base case scenario (solid lines) with most optimistic and pessimistic scenarios (dotted lines)

life year, $100 \%$ uptake of antiretroviral therapy and elective caesarean delivery makes an additional $£ 14000$ available to detect each additional infected woman, compared with the 1997 uptake of interventions. However, a decision maker assuming the most pessimistic net benefit scenario, but willing to pay up to $£ 15000$ per life year gained would spend the same sum to identify an additional maternal infection as a decision maker assuming base case net benefit and willing to pay up to $£ 10000$ per life year gained.

\section{Cost effectiveness of universal versus selective antenatal screening}

We calculated prevalence thresholds above which universal screening would be cost effective using a range of assumptions. Table 4 shows the number of districts with prevalence above the threshold. In London, all 16 districts should adopt universal testing assuming test costs of $£ 2.70$ and up to 6 minutes’ pretest discussion. Most should adopt universal testing with test costs as high as $£ 15$. Outside London, however, assuming a $£ 2.70$ test cost and 4 minutes for pretest discussion, no more than 50 districts out of 102 should adopt universal testing in preference to a selective strategy with $20 \%$ uptake and only 17 districts if a selective strategy had $60 \%$ uptake. However, if test costs were $£ 0.60$ and uptake $95 \%$, almost all districts could adopt universal

Table 3 Estimates of lifetime costs (discounted at 6\% per year) and life years (discounted at 2\% per year) of HIV infected women and their children according to whether the woman's infection was known about during pregnancy

\begin{tabular}{|c|c|c|c|c|c|c|c|}
\hline & \multicolumn{3}{|c|}{ Discounted expected costs (£s) } & \multicolumn{3}{|c|}{ Discounted life expectancy (years) } & \multirow{2}{*}{$\begin{array}{l}\text { Net benefit } \\
\text { (£s) } \dagger\end{array}$} \\
\hline & Woman* & Infant & Total & Woman* & Infant & Total & \\
\hline \multicolumn{8}{|l|}{ Base case } \\
\hline Woman's HIV infection known during pregnancy & 104344 & 6086 & 110430 & 13.959 & 37.254 & 51.213 & \\
\hline Woman's HIV infection not known during pregnancy & 79961 & 15636 & 95597 & 13.504 & 31.317 & 44.821 & \\
\hline Difference & 24383 & -9550 & 14833 & 0.455 & 5.937 & 6.392 & 49090 \\
\hline \multicolumn{8}{|l|}{ Minimum net benefit§ } \\
\hline Woman's HIV infection known during pregnancy & 117063 & 3154 & 120217 & 12.365 & 37.011 & 49.376 & \\
\hline Woman's HIV infection not known during pregnancy & 54034 & 6712 & 60746 & 11.661 & 30.536 & 42.197 & \\
\hline Difference & 63029 & -3558 & 59471 & 0.705 & 6.456 & 7.181 & 12335 \\
\hline \multicolumn{8}{|l|}{ Maximum net benefitT } \\
\hline Woman's HIV infection known during pregnancy & 104344 & 9555 & 113899 & 13.959 & 37.254 & 51.213 & \\
\hline Woman's HIV infection not known during pregnancy & 88513 & 22794 & 111307 & 13.736 & 31.317 & 45.053 & \\
\hline Difference & 14765 & -13239 & 2592 & 0.223 & 5.937 & 6.160 & 59012 \\
\hline
\end{tabular}

*From time of antenatal booking. †Net benefit assuming willingness to pay $£ 10000$ per life year gained.

łBase case assumptions: treatment efficacy base case, treatment started before AIDS but not at diagnosis, untested mother diagnosed 20 months later, base case paediatric resource use.

$\S$ Minimum benefit: treatment efficacy low, treatment started at diagnosis, untested mother diagnosed 51 months later, low paediatric resource use.

ףMaximum net benefit: treatment efficacy base case, treatment started before AIDS but not at diagnosis, untested mother diagnosed 10 months later, high paediatric resource use. 
Table 4 Number of districts in which universal screening would be cost effective compared with selective screening according to rates of uptake of testing, pretest discussion time, and test costs and assuming willingness to pay $£ 10000$ per additional life year gained

\begin{tabular}{|c|c|c|c|c|}
\hline \multirow[b]{3}{*}{ Length of pretest discussion (min) } & \multicolumn{4}{|c|}{ No of districts } \\
\hline & \multicolumn{2}{|c|}{$\begin{array}{l}\text { Test costs in } \\
\text { London } \\
\text { (16 districts) }\end{array}$} & \multicolumn{2}{|c|}{$\begin{array}{l}\text { Test costs outside } \\
\text { London } \\
\text { (102 districts) }\end{array}$} \\
\hline & $£ 2.70$ & $£ 15.00$ & $£ 0.60$ & $£ 2.70$ \\
\hline \multicolumn{5}{|c|}{ Universal uptake $\mathbf{9 5} \%$, selective uptake $\mathbf{2 0} \%$} \\
\hline 0 & 16 & 14 & 102 & 72 \\
\hline 1 & 16 & 13 & 102 & 71 \\
\hline 2 & 16 & 13 & 100 & 61 \\
\hline 4 & 16 & 13 & 95 & 50 \\
\hline 6 & 16 & 13 & 82 & 39 \\
\hline \multicolumn{5}{|c|}{ Universal uptake $95 \%$, selective uptake $\mathbf{6 0 \%}$} \\
\hline 0 & 16 & 13 & 102 & 35 \\
\hline 1 & 16 & 13 & 102 & 27 \\
\hline 2 & 16 & 13 & 100 & 22 \\
\hline 4 & 16 & 13 & 80 & 17 \\
\hline 6 & 16 & 12 & 55 & 14 \\
\hline \multicolumn{5}{|c|}{ Universal uptake $\mathbf{5 0} \%$, selective uptake $\mathbf{2 0} \%$} \\
\hline 0 & 16 & 13 & 102 & 61 \\
\hline 1 & 16 & 13 & 100 & 47 \\
\hline 2 & 16 & 13 & 92 & 37 \\
\hline 4 & 16 & 13 & 66 & 21 \\
\hline 6 & 16 & 13 & 38 & 17 \\
\hline \multicolumn{5}{|c|}{ Universal uptake $\mathbf{5 0} \%$, selective uptake $\mathbf{6 0} \%$} \\
\hline$\overline{0}$ & 0 & 0 & 0 & 0 \\
\hline 1 & 0 & 0 & 0 & 0 \\
\hline 2 & 0 & 0 & 0 & 0 \\
\hline 4 & 0 & 0 & 0 & 0 \\
\hline 6 & 0 & 0 & 0 & 0 \\
\hline
\end{tabular}

testing with up to 4 minutes' pretest discussion. Under these conditions universal testing would even be more cost effective than a selective strategy with $60 \%$ uptake, although this would allow for only 2-3 minutes' pretest discussion. If uptake of universal testing was $90 \%$ rather than $95 \%$ the time available for pretest discussion would need to be reduced by less than half a minute in low prevalence districts.

If universal testing achieved only $50 \%$ uptake, this would not necessarily affect its cost effectiveness in London. Outside London a 50\% uptake would make universal testing less cost effective than a poor $(20 \%$ uptake) selective strategy in low prevalence districts unless pretest discussion was limited to one minute. Universal testing at $50 \%$ uptake would be less cost effective than a selective programme with $60 \%$ uptake as it would cost more and identify fewer women.

Several additional findings emerged. Firstly, selective testing of high risk groups would always be more cost effective than an "on request" strategy or no testing. Secondly, increasing the uptake rate is always cost effective, as long as this does not require additional resource. Finally, 100\% uptake of interventions to reduce transmission increases net benefit of diagnosis enough to allow an extra half minute for pretest discussion in a low prevalence area.

\section{Discussion}

Previous studies in developed countries have compared universal testing to no testing rather than to selective testing. ${ }^{16-21}$ Selective testing may stigmatise women and is liable to miss many of those infected. ${ }^{21} \mathrm{In}$ the United Kingdom it has performed poorly. ${ }^{22}$ However, it is appropriate to compare universal and selective screening because selective screening is economically superior to no screening and because failure to offer testing to women known to be at higher risk is ethically and legally questionable. Furthermore, no screening has never been regarded as an option in the United Kingdom. ${ }^{4}$

Comparison with selective testing weights the results against universal testing. Our analysis is conservative in two further respects. Firstly, previous recommendations for universal testing are based on a national $^{1618}$ or regional $^{20}$ average prevalence, whereas we have referred to the entire distribution of district prevalence. We have thus required that universal screening be cost effective at an extremely low local prevalence of 3-4/100 000 rather than at the outside London average of 13/100 $000{ }^{6}{ }^{14}$ Secondly, although other studies have found that universal testing saves costs, ${ }^{16-21}$ our analysis is based on a presumption that maternal diagnosis, let alone a universal testing programme, incurs additional costs. ${ }^{6}$ Earlier studies either did not allow for additional maternal care costs at all ${ }^{1821}$ or included only the costs occasioned by earlier onset of maternal care ${ }^{1617}$ or by additional maternal life expectancy. ${ }^{20}$ None allowed for both these factors. Also, none took account of higher lifetime costs in the infected child followed from birth.

Despite these conservative features, our analysis broadly supports the recent decision by the Department of Health that HIV testing should be offered universally through the United Kingdom ${ }^{5}$ rather than only in higher prevalence areas. ${ }^{4}$ To be cost effective throughout the United Kingdom, universal testing requires test costs to be minimised; a $£ 0.60$ cost could be achieved through centralisation or by pooling sera without prejudicing test sensitivity or specificity. ${ }^{23}$

\section{Pretest discussion}

The emotionally sensitive nature of HIV testing has led to a consensus that informed consent should be obtained before testing. Our analysis shows that universal testing could not be cost effective throughout the United Kingdom if lengthy pretest counselling is instituted. However, given low test costs and uptake at $95 \%$, universal testing with 3 to 4 minutes' pretest discussion is more cost effective than a selective strategy when judged by the standards of other healthcare interventions. ${ }^{11}$

The precise time available for discussion in a universal programme depends on how well a selective strategy might perform. We assumed a $20 \%$ uptake, which is perhaps optimistic given the $8 \%$ observed rate in $1997 .^{22}$ However, even if a selective strategy achieved 60\% uptake, through improved pretest information, universal testing would still be cost effective in virtually all districts with 2 to 3 minutes' discussion. A recent study of universal testing achieved 88\% uptake with an average 2.6 minutes' pretest discussion without compromising knowledge of zidovudine or creating anxiety. ${ }^{24}$

The $90 \%$ target set by the Department of Health for the year $2002^{5}$ has essentially the same implications as the $95 \%$ assumed here, taking around 0.5 minutes off the time available for pretest discussion in low prevalence areas. However, the $50 \%$ target for the end 
Key messages

- In 1997 only 13\% of undiagnosed HIV infection in pregnant women was picked up on antenatal testing, resulting in many preventable paediatric infections

- Assuming NHS willingness to pay $£ 10000$ per life year gained, universal testing would be much more cost effective than selective testing throughout London on any reasonable assumptions on costs, prevalence, and uptake of testing

- Outside London, universal testing would also be cost effective, even allowing 2-4 minutes for pretest discussion, provided that test costs were no more than $£ 0.60$ and uptake exceeded $90 \%$

- Low cost tests could be achieved by pooling antenatal sera or centralisation of testing

- Universal testing with uptake of 50\% may be less cost effective than a well run selective programme

of 2000 may not be cost effective if sufficient time is allowed for pretest discussion. It would be more costly and less effective than a $60 \%$ uptake selective strategy.

\section{Other factors affecting cost effectiveness}

Analyses of cost effectiveness are necessarily limited in scope, and we did not include the following factors, although the direction and potential size of their effects has been discussed elsewhere ${ }^{6}$ : anxiety caused by testing, the potential of earlier diagnosis to reduce the probability that the mother will infect others, quality adjustment of life years, and the societal costs of caring for orphaned children. Currently, a keen debate revolves around the cost effectiveness of lowering vertical transmission rates still further by triple antiretroviral therapy, the merits of elective caesarean section in women on triple therapy ${ }^{25}$ and the risk of toxicity in infants exposed to antiretroviral drugs in utero. Although there was no evidence of toxicity from zidovudine in infants followed for 3-5 years, ${ }^{26}$ mitochondrial cytopathy has now been reported in eight uninfected infants exposed to antiretroviral drugs in pregnancy (S Blanche et al, second conference on global strategies for the prevention of HIV transmission from mothers to infants, Montreal, Canada, September 1-6, 1999). This debate needs to be kept under close review, but it is likely to lead to fine tuning rather than wholesale revision of net benefit estimates.

Uncertainty concerning the costs of caring for the infected mother and child, the efficacy of antiretroviral drugs, and delay in diagnosing an infected woman not identified in pregnancy were explored extensively using published material. ${ }^{6-8}$ Even on the most pessimistic view, a decision maker willing to pay $£ 15000$ per additional life year should be prepared to fund the same screening programme as a decision maker assuming the base case net benefit and prepared to pay only $£ 10000$ per life year. This lends a degree of robustness to our conclusion that universal screening would be cost effective throughout Britain as long as test costs can be kept to $£ 0.60$ in low prevalence areas and uptake kept above $90 \%$.

We thank our colleagues in the HIV surveillance team at the Institute of Child Health and in national surveillance centres (Communicable Disease Surveillance Centre, Public Health Laboratory Service, Colindale, and the Scottish Centre for Infection and Environmental Health, Glasgow).
Contributors: DMG was the principle grant applicant and provided clinical input. MJS developed the decision model for screening with AEA, and both have independently programmed it. The analyses presented here were developed by AEA assisted by DMG. JR and RG both contributed to development of the component modules within the analysis (epidemiology, reduction of mother to child transmission, paediatric and maternal disease progression and costs). All authors contributed to interpretation of results, and commented on the final draft. AEA is the guarantor.

Funding: Department of Health.

Competing interests: None declared.

1 Gibb DM, MacDonagh SE, Tookey PA, Duong T, Nicoll A, Goldberg DJ, et al. Uptake of interventions to reduce mother-to-child transmission of HIV in United Kingdom and Ireland. AIDS 1997;11:F53-8.

2 Connor EM, Sperling RS, Gelber R, Keslev P, Scott G, O'Sullivan MJ, et al. Reduction of maternal-infant transmission of human immunodeficiency virus type 1 with zidovudine treatment. Pediatric AIDS Clinical Trials Group Protocol 076 Study Group. N Engl J Med 1994;331:1173-80.

3 International Perinatal HIV Group. Mode of delivery and the risk of vertical transmission of human immunodeficiency virus type1. A meta-analysis of fifteen prospective cohort studies. $N$ Engl J Med 1999;340:977-87.

4 Department of Health. Guidelines for offering voluntary named HIV antibody testing to women receiving antenatal care. London: HMSO, 1994. (PL/CO(94)5.)

5 Department of Health. Reducing mother to baby transmission of HIV. London: Stationery Office, 1999. (Health service circular 1999/183.)

6 Ades AE, Sculpher M, Gibb D, Ratcliffe J, Gupta R. A cost-effectiveness analysis of antenatal HIV testing in the UK. Uxbridge: Health Economics Research Group, Brunel University (in press). (Research report No 26.)

7 Gibb DM, Ades AE, Gupta R, Sculpher M. Costs and benefits to the mother of antenatal HIV testing: estimates from simulation modelling. AIDS 1999;13:1569-76.

8 Sculpher M, Gibb D, Ades AE, Ratcliffe J, Duong T. Modelling the costs of paediatric HIV infection and AIDS: comparison of infected children born to screened and unscreened mothers. AIDS 1998;12:1371-80.

9 Ratcliffe J, Ades AE, Gibb D, Sculpher M, Briggs A. Prevention of motherto-child transmission of HIV infection: alternative strategies and their cost-effectiveness. AIDS 1998;12:1381-8.

10 Stinnett AA, Mullahy J. Net health benefits: a new framework for the analysis of uncertainty in cost-effectiveness analysis. Med Decis Making 1998; 18:S68-80

11 Department of Health. Register of cost-effectiveness studies, 1994. London: Department of Health, Economics and Operational Research Division, 1994

12 Gibb D. Guidelines for management of children with HIV infection. Horsham: AVERT, 1998.

13 Raleigh VS, Kiri VA. Life expectancy in England: variations and trends by gender, health authority, and level of deprivation. J Epidemiol Community Health 1997;51:649

14 Ades AE, Gupta R, Gibb DM, Duong T, Nicoll A, Goldberg DJ, et al. Selective versus universal antenatal HIV testing: epidemiological and implementational factors in policy choice. AIDS 1999;13:271-8.

15 Department of Health. Policy appraisal and health. London: DoH, 1995.

16 Mauskopf JA, Paul JE, Wichman DS, White AD, Tilson HH. Economic impact of treatment of HIV-positive pregnant women and their newborns with zidovudine. Implications for HIV screening. JAMA 1996;276:132-8

17 Ecker JL. The cost-effectiveness of human immunodeficiency virus screening in pregnancy. Am J Obstet Gynecol 1996;174:716-21.

18 Gorsky RD, Farnham PG, Straus WL, Caldwell B, Hottgrave DR, Simonds $\mathrm{RS}$, et al. Preventing perinatal transmission of HIV-costs and effectiveness of a recommended intervention. Public Health Rep 1996;111:335-41.

19 Lewis R, O'Brien JM, Ray DT, Sibai BM. The impact of initiating a human immunodeficiency virus screening program in an urban obstetric population. Am J Obstet Gynecol 1995; 173:1329-33.

20 Postma MJ, Beck EJ, Mandalia S, Sherr, L, Watters MDS, Houweling H, et al. Universal HIV screening of pregnant women in England: cost effectiveness analysis. BMJ 1999;318:1656-60.

21 Stoto MA, Almerio DA, McCormick MC. Reducing the odds. Preventing perinatal transmission of HIV in the United States. Washington, DC: National Academy Press, 1998:397.

22 Tookey PA, Gibb DM, Ades AE, Duong T, Masters J, Sherr L, et al. Performance of antenatal HIV screening strategies in the UK. J Med Screen 1998;5:133-6.

23 Parry JV, Mahoney A, Mortimer PP. Are seroepidemiological surveys for human immunodeficiency virus infection based on tests on pools of serum specimens accurate and cost-effective? Clin Diag Virol 1993;1:167-78.

24 Simpson WM, Johnstone FD, Boyd FM, Goldberg DJ, Hart GJ, Gormley SM. A randomised controlled trial of different approaches to universal antenatal HIV testing: uptake and acceptability. Annex: Antenatal HIV testing-assessment of a voluntary approach. Health Technology Assessment $1999 ; 3$.

25 Stringer JS, Rouse DJ, Goldenberg RL. Prophylactic cesarean delivery for the prevention of perinatal human immunodeficiency virus transmission. JAMA 1999;281:1946-9.

26 Sperling RS, Shapiro DE, McSherry GD, Britto P, Cunningham BE, Culnane M, et al. Safety of the maternal-infant zidovudine regimen utilized in the Pediatric AIDS Clinical Trial Group 076 Study. AIDS 1998;12: 1805-13.

(Accepted 24 September 1999) 